\title{
Paradoxical prosopagnosia in semantic dementia
}

\author{
Sven-Erik Fernaeus ${ }^{{ }^{*}}$, Per Östberg ${ }^{2}$, Lars-Olof Wahlund ${ }^{1}$, Nenad Bogdanović ${ }^{1}$ \\ ${ }^{1}$ Department of Neurobiology, Care Sciences and Society, Karolinska Institutet, Stockholm, Sweden; \\ *Corresponding Author: per.ostberg@ki.se \\ ${ }^{2}$ Department of Neuroscience, Uppsala University, Uppsala, Sweden
}

Received 29 October 2012; revised 30 November 2012; accepted 7 December 2012

\begin{abstract}
Objective: To study episodic and semantic memory for faces and other non-verbal information in semantic dementia (SD). Background: Semantic memory impaired in the rare diagnosis of SD by definition, including knowledge about wellknown persons and their appearance. Episodic memory is held to be better preserved. Methods: Two computerized face recognition tests were administered, one measuring episodic memory (Male Faces) and one semantic memory (Political Faces) in addition to a comprehensive test battery. A computerised test of non-verbal semantic memory for national symbols (Euro Flags) was also administered druring the retention interval of the Male Faces test. Results: The SD participants were severely impaired in the episodic face recognition test. In contrast, their performance was in the normal range in Euro Flags and Political Faces, based on knowledge of national political figures. Conclusion: The results are discussed in terms of preserved dynamic memory and severely impaired memory for static facial information in semantic dementia. Research proposals regarding further studies of this paradoxical prosopagnosia in semantic dementia are presented in order to clarify issues regarding static versus dynamic aspects of face memory.
\end{abstract}

Keywords: Face Recognition; Semantic Dementia; Prosopagnosia; Temporal Atrophy

\section{INTRODUCTION}

Face recognition is generally held to be a specializetion within the human visual system. The ability to recognize faces may deteriorate with normal ageing as early as around age $50[1,2]$. Usually, however, prosopagnosia or impaired recognition of familiar faces results from acquired brain damage. Prosopagnosia is thus a prominent feature of semantic dementia (SD), a clinical syn- drome caused by atrophy of the temporal poles, and anterior and inferior temporal lobe neocortex. SD patients gradually lose the ability to understand meaning, that is, progressive semantic memory loss, whereas episodic memory and spatial cognition are better preserved $[3,4]$.

The exact nature and severity of the prosopagnosia in SD are unclear. It is not clear whether it occurs only in bilateral symmetric temporal atrophy, in predominantly right-hemispheric atrophy, or is a general marker of SD. It may be a secondary feature in the more common left hemispheric cases [5]. According to one study, SD patients with predominant left temporal atrophy show preserved recognition of famous faces despite strongly impoverished factual knowledge of the persons depicted [6]. This ability was impaired, however, if different photographs of the celebrities were used at study and test, suggesting differential contribution from semantic memory and basic perceptual mechanisms in face perception. Moreover, an ambiguity in face recognition has been reported, with preserved recognition of an "iconic" politician (Silvio Berlusconi) in an SD patient who was otherwise unable to recognize even highly familiar faces [7]. One may thus ask whether the prosopagnosia in SD is linked primarily to perceptual and encoding difficulties, or is a genuine semantic memory disturbance like the core signs of such as semantic anomia. The aim of the present study was to compare the performance of SD patients in a relatively difficult and thoroughly developed episodic face recognition test with their performances in related nonverbal semantic memory tests.

\section{Functional Anatomical Considerations}

The basic pathological feature of SD is neuronal loss in the cerebral cortex with topical and laminar selection and accentuation. The anterior temporal lobes and cortical layer II are particularly affected. The neurons of layer II are small pyramidal neurons that project mainly to neighboring areas in the temporal cortex. They are the place of origin for $\mathrm{U}$ projections, i.e. projections from gyrus to gyrus. Together with layer III neurons, they are also the source of feedforward projections. This indicates that information directed to hierarchically higher regions 
originate from these neurons as well as from the neurons in the layer III. The difference is that layer III neurons project to more remote areas as well to the commissural areas in the contralateral hemisphere. It is very likely that degeneration waves spread through short associative projections, disconnecting regions that are lower in the functional and morphological hierarchy. Atrophy in SD predominantly affects the temporal anterior region. Since layer II neurons are lost selectively, the affected areas are disconnected from adjacent areas but not from remote ones. This implies that areas within the anterior temporal region are disconnected locally, but not from the occipito-temporo-parietal region known as a polymodal sensory area. The affected areas are situated around the entorhinal zone and project with short feedforward projections to the hierarchically higher entorhinal cortex. In this cortex, input converges from all cortical regions and subcortex as well. Thus, input dependent on the parietooccipital-temporal border is preserved but not input from the temporal anterior cortex. SD therefore involves a selective alteration of anatomy and function.

How might this alteration affect face recognition? An obvious but in no way trivial fact is that face recognition tests do not actually employ faces as stimuli. Instead they use photographs of faces, i.e. static (and silent) two-dimensional representations of faces. Since faces most often are characterized by movement, not least in speakers, it is possible that the exclusion of dynamic facial features affects face recognition in SD adversely. As explained above, the remote connections to the parieto-occipitotemporal region are preserved in SD, and motor kinetic input from the frontal cortex is projected there. Possibly, then, SD patients may be unable to recognize static facial pictures presented to them in short durations, owing to devastation of the short connections between entorhinal and "prosopagnostic" areas in the anterior temporal lobe. Faces encoded via dynamic pictures, such as in television, would on the other hand rely more on the preserved interaction between frontal cortex and posterior polymodal cortex. This is speculation, and further anatomical explanation is needed.

The selectivity of pathology in SD is also apparent in the selective affection of the dentate gyrus of the hippocampal complex. This affection is readily visualized on neuropathological examination as ubiquitin-positive inclusions in the neurons. Selective pathology of the dentate gyrus can further interfere with face memory processes, since neuronal input has to pass the dentate gyrus during encoding. The dentate gyrus is directly connected with the entorhinal cortex and transmits impulses back there indirectly via the CA-subiculum region. We suggest that neurons of the dentate gyrus are selectively affected, and that this selectivity is part of the selective cortical pathology.
To conclude, SD is pathologically a highly selective disorder in which a specific group of cells and projections are affected, especially in short, face-to-face projections, whereas remote ones are spared at least initially. This pathological pattern may explain some of the neuropsychological phenomena seen in SD but not in AD.

\section{METHOD}

\subsection{Preparation of Computerized Tests}

An episodic memory test, Male Faces, was developed with the initial purpose of differentiating patients being examined for memory problems. A second aim of the test development was that Male Faces should still be able to detect high levels of face recognition ability among healthy individuals with the ultimate goal of reducing ceiling effects in control samples. The production criteria for the test, were the following: 1) Targets and distractors should be as similar as possible regarding structural facial traits; 2) Irrelevant information (clothing, glasses, etc.) should be erased from photographs; 3) All faces should have neutral expressions (no smiles or other emotional expressions); 4) Only male faces should be included owing to the well-known interaction between viewer's gender and face photo's gender [8], according to which female viewers recognize female faces better than male faces, whereas male viewers do not show gender-dependent face memory; 5) Age attributes/variation should be kept to a minimum without facilitating performance in any sex. From an initial sample of 900 photographs of police students, all photographs of faces that did not meet criteria 3 - 4 above were excluded. Among the photographs that remained, three raters compared the faces and tried to form as many similar looking pairs as possible. After this pairing provisional sets of targets and distractors were formed (A-B, C-D, E-F), which resulted in subsets with 25 - 30 pairs in each. Then raters met and excluded those pairs with least resemblance until a consensus was reached regarding 21 pairs in each subset. After scanning, each photograph in the subsets was edited using the Photoshop package. Thus clothing (collars and ties) was erased from each photograph as well as signs of acne or wounds. Other dots were also erased in order to reduce focal details. If there was little variation in hairstyle or hair colors in one subset compared to the others, some photographs were exchanged between sets. If faces deviated in size, they were resized in order to fill about the same area of the $5 \times 8 \mathrm{~cm}$ picture area. All faces were grey scale.

Using PhotoShop, HyperCard and the REALBasic packages, the computerized test was developed on the MacOS platform and using REALBasic it was compiled for both MacOS and Windows using the same presentation parameters, the same response keys, and the same time 
measuring methods. The computer used at clinical testing was an Apple Powermac 7100 with a 17" screen. In the testing of healthy controls PCs were used. At presentation the test person was instructed to concentrate on the middle of the screen and try to remember each face. Faces were shown for $5 \mathrm{~s}$ with a brief interstimulus interval (ISI) of one second.

After presentation of the 21 photographs the test person had to perform a test for national symbols, Euro Flags. The test is a nonverbal semantic memory test based on 24 photographs of flags. Twelve target photos show European flags including the European Union flag, and the reminding twelve distractor photos show Asian, American, African or other non-European flags. The task is to press the "Yes button" if a European flag is shown, otherwise the "No button". The target flags represent Sweden, Norway, Finland, Denmark, Germany, United Kingdom, France, Spain, Italy, Greece, Switzerland and the European Union. Each of the distractor flags is similar to one of the European flags in color or structure or in both. As an example the flag of Palau, one of the distractors, is similar to the Swedish flag in colour, but not in form, having a yellow circle instead of a yellow cross on the blue background.

This intermediate test, filling the retention interval for Male Faces, took about 4 minutes, after which the test phase of the Male Faces test was run. The test person was instructed to press a button, "Yes" (left arrow) if the face had been shown earlier and "No" (right arrow) if the face had not been shown at presentation. The reaction times (RT) were measured in microseconds and recalculated into milliseconds. Mean reaction times were saved as well as the proportion of hits, false alarms, misses and correct rejections. On the basis of each of these proportions percent correct was calculated including both hits and correct rejections. In this report only percent correct will be reported owing to differences in calculating RT between the different computerised versions.

The Political Faces test is a nonverbal semantic memory test based on 24 photographs of members of the Swedish parliament during 1998. Twelve photos show the male parliament members most often seen on the national TV channels and nationwide distributed newspapers during the years preceding the date of testing. The remaining twelve photos show members of parliament never or very seldom seen in the same media. Care was taken that the politicians came from all over the country representing all parties of the parliament. Most of the party leaders and the most well known members of the Swedish government at the time of testing appeared on the target photos. Test persons should check the "Yesbutton", the left arrow key, if they recognized the person shown as a known national politician and the "No-button”, right arrow key, if the person shown would be re- garded as unknown. Participants were not required to say the names of the known politicians, but were asked to press the response buttons quickly. Known and unknown faces were distributed in a random fashion.

It may be noted that, for some test persons, the Political Faces test may be experienced as a test of incidental learning. This is due to the fact that the learning period preceding the semantic memory stage may have been much shorter than in the case of learning European flags, resulting in a less stable knowledge structure than what is usually expected in semantic memory. The visual appearances of the national flags used in the Euro Flags test have not changed during the last decades, while the upper strata of political parties at least in Sweden undergo changes at least every fourth year. Among the 12 original known politicians in the 1998 version of the test at least two are out of the media focus seven years later-although still known by the older public - they are probably unknown to younger subjects in their late teens or early twenties, which implies that the same stimuli may not be used if a replication of the study would be made today. However, because of travel habits or sheer differences in interest, the same may hold for some of the test persons regarding the Euro Flags test. This means that knowledge of some of the flags may actually be the result of recently acquired incidental learning, rather than held and stable knowledge in semantic memory.

The interpretation of the entire set of stimuli in both the Political Faces and Euro Flags as pure tests of nonverbal semantic memory must therefore be viewed with some caution. The term nonverbal tests of semantic memory will nonetheless be used in the present study for both of these tests, because most of the items for the absolute majority of the participants in the study, including healthy controls, were part of well-established knowledge about the visual appearances of nation symbols and prominent national politicians; they were not learned or encountered during just one single memory encoding occasion.

Among nondemented normal range for the Political Faces test is 83\% - 100\% (20 - 24) and for the Euro Flags test $79 \%$ - 96\% (19 - 23). Because only middle-aged or older subjects have been tested so far, data on age differences and developmental effects are not yet available.

Blood flow in frontal, temporal, parietal and occipital lobes was measured using SPECT, and magnetic resonance imaging (MRI) was performed on each patient according to routine procedures at the Memory Clinic of Huddinge University Hospital [9].

\subsection{Participants}

Four samples of healthy individuals participated in the test construction phase of the study: senior high school students $(\mathrm{N}=31)$, college students, police officers $(\mathrm{N}=$ 
30), and middle aged and elderly ( $\mathrm{N}=32)$, in sum 131 healthy controls.

All SD cases $(\mathrm{N}=5)$ available for examination at Huddinge University Hospital during one year were included in the study. Figure 1 shows left temporal lobe atrophy on a coronal MRI scan of one of the patients, and Figure 2 shows a similar scan of a right temporal lobe case. All five cases had similar atrophy in these regions. The assessment for dementia was based on a comprehensive neuropsychological test battery as well as MRI, SPECT and EEG, see Table 1 for patient characteristics. SD was diagnosed according to international consensus criteria [10]. The subjects are described more closely below.

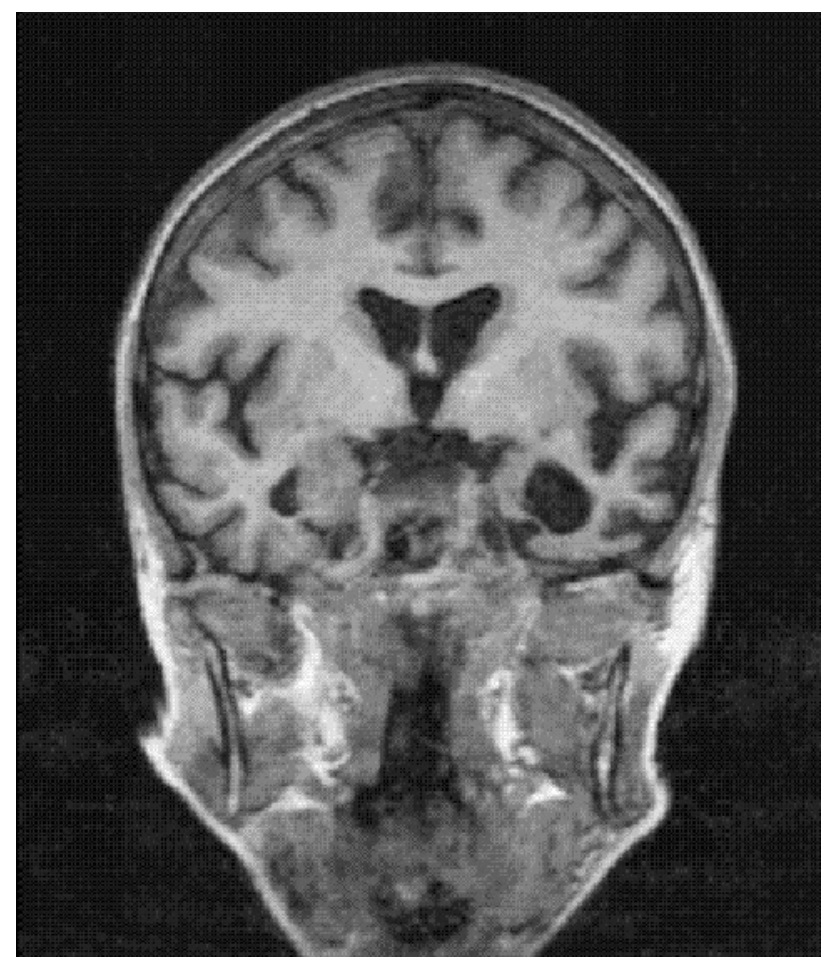

Figure 1. A case of semantic dementia with severe left lateral temporal lobe atrophy, female 60 years.

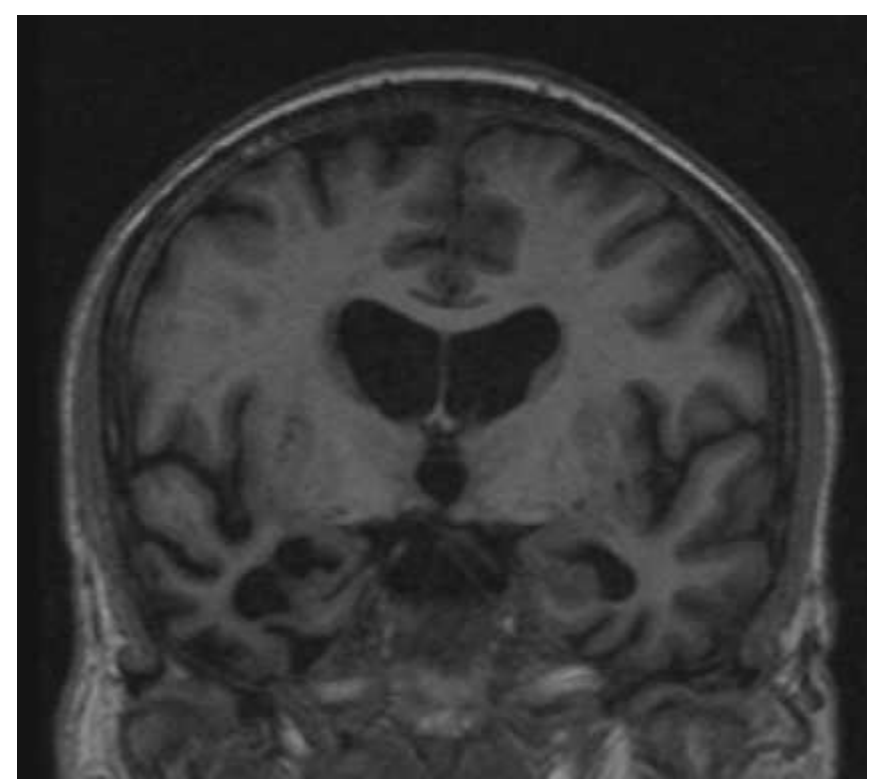

Figure 2. A case of semantic dementia with severe right lateral temporal lobe atrophy, male 65 years old. 
Table 1. Patient scores in the regular test battery at the memory clinic. Tests of semantic memory and episodic memory tests involving concrete nouns are italicized. Subnormal scores are boldface.

\begin{tabular}{|c|c|c|c|c|c|c|}
\hline Case & 1. FA & 2. IN & 3. BT & 4. SC & 5. $\mathrm{RH}$ & Norm \\
\hline Age/Gender & $65 / M$ & $60 / \mathrm{M}$ & $62 / F$ & $60 / \mathrm{F}$ & $65 / M$ & $65.1 \pm 6.6$ \\
\hline Education & 15 & 8 & 15 & 14 & 12 & $11.2 \pm 3.4$ \\
\hline EEG & $\begin{array}{c}\text { Normal } \\
9 \mathrm{~Hz} \\
\text { LT }\end{array}$ & $\begin{array}{l}\text { Normal } \\
10 \mathrm{~Hz}\end{array}$ & Normal & & FT & \\
\hline SPECT & $\begin{array}{l}\text { Strongly } \\
\text { Reduced }\end{array}$ & RT & LT & LT & RT & \\
\hline MRI & $\begin{array}{c}\text { Bilateral } \\
\text { L > R }\end{array}$ & & & & & \\
\hline MMSE & 24 & 25 & 24 & 25 & 28 & 29 \\
\hline Boston Naming Test & 5 & 5 & $<20$ & 2 & $<20$ & $54.5 \pm 4.3$ \\
\hline WAIS-Similarities & 5 & 3 & 2 & 0 & 14 & $22.1 \pm 3.4$ \\
\hline Information & 7 & 1 & 3 & 9 & 17 & $23.7 \pm 3.0$ \\
\hline WAIS-Block Design & 24 & 28 & 30 & 22 & 32 & $30.9 \pm 6.8$ \\
\hline SRB-2 & 25 & & 24 & & 12.25 & $20.6 \pm 4.1$ \\
\hline WAIS-Digit Span & 15 & 15 & 12 & 10 & 12 & $12.5 \pm 5.4$ \\
\hline Corsi Block Tapping & 5 & 5 & 5 & 5 & 7 & $5 \pm 0.5$ \\
\hline WAIS-DigitSymbol & 38 & 18 & 38 & & 37 & $43.7 \pm 9.8$ \\
\hline GC-Free Noun Recall & 2 & & 2 & 3 & 4 & $6.2 \pm 2.4$ \\
\hline GC-Free Recognition & 2.35 & & & 0.24 & 2.35 & $3.3 \pm 1.1$ \\
\hline Rey-AVLT-1 & 5 & 2 & 3 & 6 & 4 & $6.0 \pm 1.9$ \\
\hline Rey-AVLT-5 & 9 & 5 & 7 & 10 & 11 & $11.9 \pm 2.5$ \\
\hline TMT-A RT & 48 & 70 & 46 & 93 & 51 & $40.7 \pm 14.8$ \\
\hline TMT-B RT & 74 & 187 & 156 & 186 & 84 & $94.5 \pm 41.3$ \\
\hline Letter fluency FAS & & 13 & 25 & 21 & & \\
\hline Category Fluency & & 3 & 0 & 1 & & \\
\hline
\end{tabular}

Note: Norms are based on an age matched sample $(\mathrm{N}=42)$ of healthy controls with a mean IQ of 107 , a mean age of 65.1 , range 50 - 75 , and tested at the memory clinic, Karolinska university hospital, Huddinge.

Case 1: SD with predominant left-hemisphere atrophy

This 65-year-old ambidextrous man with a bilingual background (Swedish, German) presented with increasing difficulties in "remembering" the names of animals, plants, and well-known people. At the clinical interview, he was courteous, well groomed, and talkative. Spontaneous speech appeared normal and he was orientated in time and place. Visuoconstructive skills and verbal and non-verbal working memory were within normal range, and non-verbal reasoning was at a superior level. He performed Weigl's sorting test without trouble. In contrast, his performance on tasks involving thing concepts (e.g. picture naming, definitions, generating concrete nouns) was impaired. He showed a pronounced semantic anomia, notably for animals, vegetables, and famous faces. Associative agnosia was present (Pyramids and Palm Trees Test, three-picture version). Repetition was preserved. Syntactic comprehension was preserved in both
Swedish and German (Bilingual Aphasia Test). Reading aloud showed a surface alexic error pattern. Spelling on dictation showed a lexical agraphic pattern. Repeated testing indicated further deterioration of conceptual knowledge and slowing in attention-demanding tasks, while spatial cognition was retained. When last seen at the clinic, his anomia was severe. He referred to every oblong inanimate object as a "tamp" (rope's end; he had been a yachtsman), whereas animals and humans alike were referred to as "a young woman" or "a man".

His EEG was normal. Alpha rhythm with peak frequency at $9 \mathrm{~Hz}$. MRI scanning showed marked bilateral but asymmetric temporal lobe atrophy, more pronounced at the left side and anteriorly. SPECT revealed strongly reduced left temporal perfusion.

Case 2: SD with predominant right-hemisphere atrophy

This 60-year-old right-handed man presented with increasing difficulties in understanding words and pictorial 
symbols. Family members reported a tendency towards behaving embarrassingly in larger social contexts. He still worked part-time in a technically demanding position, but withdrew from verbally taxing duties because of his inability to understand words. At the clinical interview he was well groomed, courteous, and humorous. He was cooperative and claimed to be eager to learn why he had become "crazy, or rather, defective”. Jocular and compulsive traits were shown at the ward. For instance, he supervised the dishwasher so that nobody put the dishes in another order than that preferred by him. He expressed the concern that he might be a nuisance to the staff. Neuropsychological testing showed preserved working memory, visuoconstructive praxis, and psychomotor speed, while verbal abilities and identification of visual symbols were strongly impaired. Memory for faces, both known and unknown, was also strongly impaired. At speech-language assessment, he showed a profound semantic anomia and inability to understand virtually any concrete noun. In contrast, his spoken output was phonologically and syntactically well formed. Surface alexia and lexical agraphia were presented, as was associative agnosia.

Case 3: SD with predominant left-hemisphere atrophy

This 62-year-old right handed woman presented with increasing difficulties in finding nouns even for very common objects. Her language problems had started 6 years before testing. She took up painting and participated in semi pro shows with her art and managed to go abroad doing this in spite of her severe language problems. Everyday activities like shopping did not trouble her, as she found out that she could make small drawings of items to be bought at the grocery. However, she was unable to make a drawing from memory, and was dependent on having an item in front of her when producing these small drawings. At the clinical interview she was anxious to find an explanation for her impairment, and she offered several hypotheses for her impaired speech. At testing she was cooperative and responded quickly to questions. Her personality was rather impulsive. Judging from her scores on AMQ, a self-report questionnaire [10], subjective memory for places as well as some aspects of face memory were preserved. Neuropsychological testing showed preserved logical and visuospatial skills and impaired verbal learning. Speechlanguage assessment showed profound anomia on picture naming and severely impaired letter and category fluency.

Case 4: SD with left hemisphere atrophy

This 60 year-old right handed woman presented with increasing speech difficulties. Neuropsychological testing showed severely impaired semantic memory, impaired verbal learning and verbal episodic memory. Personal episodic memory and working memory for digits were intact as well as logical and visuoconstructive abi- lity. Speech-language assessment revealed severe semantic anomia, orthographic agraphia and surface alexia. Associative agnosia for faces and objects was presented.

Case 5: SD with right hemisphere atrophy

This 65-year-old man presented with increasing personality changes including impaired judgement. Neuropsychological testing showed impaired semantic memory and verbal learning as well as subnormal verbal episodic memory, although his verbal recognition score was preserved. His visuoconstructive ability was good as well as his Mini mental state.

For details regarding regular test scores for the SDparticipants, see Table 1.

\section{RESULTS}

\subsection{Control Samples}

In order to collect data on the performance of healthy subjects in different age groups in the Male Faces test, four groups were tested: senior high school students and college students in a medium sized town in the western part of Sweden, police officers from a big city in western Sweden, and a random sample of middle aged and elderly from a northern Swedish town. Mean scores in the Male Faces and Euro Flags tests are shown in Table 2. Because there were no significant differences between men and women in either test, separate mean scores for the two genders are not reported.

Among the group of middle aged and elderly, range 38 94, there was a significant correlation between Male Faces recognition scores and age $(r=0.58, P<0.01)$. Because the relationship was rather curvilinear, an exponential regression was better fit to the data. The nonlinear correlation was $(r=0.60)$. There was also a signifycant correlation between Euro Flags and Male Faces in this sample $(r=0.46, P<0.01)$, indicating a shared variance of about $21 \%$ between the two tests in this sample.

\subsection{SD Patients}

As can be seen from Table 3 all five SD patients perform at a random level in Male Faces with a median of 55 percent correct, while four of them performed within the normal range in Euro Flags and Political Faces. The reversed distribution of SD cases who passed or failed the Political Faces and the Male Faces tests was signifi cant (Fisher's exact $P>0.01$ ).

\subsection{Test Behavior}

Three of the SD patients were predominantly impulsive indicated by short reaction times in the computerized tests. One of these three patients at two occasions enacted his ideas: for instance, to make it clear he was 
Table 2. Means and standard deviations in male faces, Euro flags and political faces among healthy control samples.

\begin{tabular}{ccccccccc}
\hline & & \multicolumn{2}{c}{ Male Faces } & \multicolumn{2}{c}{ Euro Flags } & \multicolumn{2}{c}{ Political Faces } \\
\hline Group of healthy controls & Median Age & $\mathrm{n}$ & Mean & Std dev & Mean & Std dev & Mean & Std dev \\
Senior high school & 18 & 38 & 80.95 & 9.62 & 83.77 & 8.60 & \\
College students & 26 & 31 & 77.16 & 7.30 & 83.45 & 8.36 & \\
Police officers & 32 & 30 & 83.89 & 7.67 & 84.03 & 6.39 & \\
Elderly & 62 & 32 & 72.33 & 14.5 & 79.95 & 8.68 & 8.72 & 88.29 \\
Middle-aged & 55 & 28 & 79.20 & 7.02 & 85.47 & 6.46 \\
\hline
\end{tabular}

Table 3. Recognition scores in non-verbal computerized memory tests among five SD participants. Subnormal scores are in boldface.

\begin{tabular}{ccccccc}
\hline Patient & Sex & Atrophy & Age & Euro Flags & Political Faces & Male Faces \\
\hline 1. FA & M & RT & 65 & 87.5 & 92 & 55 \\
2. IN & M & LT & 60 & 50 & 50 & 55 \\
3. BT & F & LT & 62 & 79 & 87.5 & 55 \\
4. SC & F & LT & 60 & 79 & 95 & 50 \\
5. RH & M & RT & 65 & 83 & 92 & 61 \\
& Median & & 62 & 79 & 92 & 55 \\
\hline
\end{tabular}

not "like the rest of you" he turned around as he remarked "Just so that you know I am a little whimsy!" (sv. snurrig)

After the testing session, one of these SD patients asked the examiner how the photos in the tests had been edited. After an explanation, and to give the patient a clue about the editing process, she was allowed to play with the Photoshop program which had been used at the design phase. After a brief introduction and with no prior experience in computer-aided painting, she quickly mastered the software and completed an expressive picture in a few minutes. This was in sharp contrast to her inability in drawing, because while her copying was preserved, her drawing from memory was severely impaired. She was unable to draw a bicycle from memory, though she was an eminent semi-pro expressive painter.

\section{DISCUSSION}

In this study, five patients with SD underwent two tests of face recognition, Male Faces and the Political Faces. The former test is an episodic face recognition test measuring recent face recognition, and the latter is conceived as a semantic face recognition test. The Male Faces test was administered to different groups showing about the same level of performance for participants below 70 years of age, which made it an appropriate instrument for the group of relatively young SD patients, who by the time of testing had a median age of 62. During the retention period of Male Faces all participants including the SD cases also took the Euro Flags test, a non-facial semantic recognition test.

SD subjects performed within normal range on the non-verbal semantic tests (Political Faces and Euro Flags) whereas episodic face recognition (Male Faces) was severely impaired. This is a surprising finding inasmuch as SD is generally characterized by severe semantic loss and clinically preserved episodic memory. This counterintuitive finding indicates that item knowledge of current politicians based on incidental encoding may in fact be preserved in SD. Performance on a semantic memory test may thus paradoxically be normal in SD. This in turn sheds doubt on the common notion of semantic memory as an atemporal form of memory, because the temporal range of "current nationwide politicians" may be 5 - 10 years, depending on the number of exposures. This may be compared to schematically organised knowledge acquired one week or a month earlier, such as knowledge about the immediate work environment and personal schedules and events tied to these. This latter kind of knowledge is, based on anecdotal clinical evidence, relatively preserved in SD, because the patients remember their appointments with examiners at the clinic and are able to run their own everyday life.

Four out of five of the SD patients also scored within normal range on the Euro Flags test, a test of non-facial semantic memory. Again, the notion of an atemporal semantic memory is called into question. Knowledge of the visual appearance of flags probably goes back far longer than knowledge of current political figures in these patients. Thus, the Euro Flags test may be regarded as a more specific semantic memory test than the Politi- 
cal Faces test. Moreover, because nation flags are conventional symbols of countries or nations, they carry more meaning and are thus more semantic in character than faces of politicians. The label semantic memory test therefore would be more appropriate for Euro Flags. Nevertheless, personal interest may play an important role in determining the number of incidental learning occasions regarding European flag knowledge. For example, flag learning may differ substantially between different groups. Irrespective of this, the artifactual schematic design of flags makes them quite different as visual objects compared to faces. The face is inherently a potential origin of a large amount of human signals or messages, whereas a nation flag does not really convey any message at all apart from signifying a nation. When analyzing what part of the world of meaning that our SD patients have lost, we may thus conclude that they may still master such non-verbal representations of central world knowledge as exemplified by flags.

The same SD patients, however, scored in the subnormal range, actually within the random range, on the Male Faces test. This is an episodic recent memory test, which, regarding the performance of the SD patients, indicates poor encoding of static person information of a non-verbal kind. Judging from the results of the study, a single presentation for 5 seconds is not enough to form even a slight recent episodic memory in these patients, even if the medial temporal lobe and more posterior cortical structures might support learning when presentation time is long and repeated. However, when the latter conditions are met, as is the case with well-known political figures who often appear as dynamic information on television, patients with SD seem to be able to form and retain robust face recognition. We may also note that $3 / 5$ of SD patients in this study had left hemispheric atrophy, not the right temporal lobe atrophy typical of semantic dementia presenting with progressive prosopagnosia.

Because of their specific problems our SD patients did not recognize celebrities of the 1960s, which is a typical finding [10]. For example, one of our patients, who was a classmate of a very famous Swede, did not recognize this national celebrity from a photo at testing. In spite of this, he incidentally mentioned that he had seen the former classmate at the airport recently, but was too shy to approach him. This is in accordance with our theory that movement properties of persons, or in other words object properties of activities involving people as objects, may aid face recognition in SD Arguably, these properties should not be viewed as part of core conceptual structures or semantic memory per se, but rather as parts of spatial structures connected to word meaning [11]. In contrast to $\mathrm{AD}$, the brain systems critical for this aspect of spatial cognition are known to be largely preserved in SD. While there is a moderate generalized brain atrophy in $\mathrm{SD}$, the region with accentuated atrophy does not involve the posterior superior temporal region or inferior parietal lobule. This characteristic pattern of atrophy was figured already by Onary \& Spatz [12].

According to James \& Gauthier [13] the posterior superior temporal sulcus, which is known to respond to motion, was "preferentially activated by objects associated with 'action' features (e.g., hops)”. This finding supports the hypothesis of preserved action cues or motion affordances in SD. Tyler et al. [14] reported activation of the left fusiform gyrus and the superior and middle temporal cortex during retrieval of object names and their associated actions. This was claimed to be consistent with the notion that tool words and animal concepts "implicitly activate the actions associated with them". However, it may also be the other way around: Because objects are often significant or required parts of actions and the conceptualization of actions and activities as such, it would not be surprising that thinking about objects and actions activates approximately the same set of brain regions.

A recent activation study by Kable $e t a l$. is very much in line with this reasoning [15]. They reported activation of areas close to MT/MST on the left "within the aspect of the middle and superior temporal gyri" when subjects were accessing knowledge of actions through words. They proposed that "the lateral occipital-temporal cortex contains a mosaic of neural regions that processes different kinds of motion, ranging from the perception of objects moving in the world to the conception of movement implied in action verbs". According to Kable et al. the "lateral occipital-temporal cortex mediates the perceptual and conceptual features of action events". However, "knowledge of actions" implies an idea of activity as such, that is, in Aristotelian terms, the potentiality for action of a person or animal. Activity may thus be mediated by relatively posterior regions, while its counterpart, action, as the actualization of activity, may be more dependent on frontal structures, which are important for initiating, maintaining, modifying, inhibiting or other properties related to action.

A methodological issue when assessing prosopagnosia is how to measure it in a clinical setting because of technical problems without making too much violence on ecological validity. Spoken words and written text are by definition much easier to expose to the patient, because they occur as such in the stimulus material. People and faces in experimental studies usually appear as pictorial representations, implying that investigators are most often confined to static images like photographs or drawings. When we design tests for the measurement of face recognition we also, in the name of perfect experimentation technique, try to refine the stimulus material in such a way as to clean them from emotional attributes. This was also the case in this study, for pure psychometric reasons, in order to arrive at a reliable and valid test. 
Photographs used in the Male Faces test were carefully chosen in order to eliminate confounding effects due to imbalance between targets and distractors. However, because people in the real world do not appear as static photographs without any emotional expressions or other motion attributes, we would rather characterize the Male Faces test, like all other alleged face memory tests, as $a$ static non-emotional face recognition test. This point must be made in this context because we might have arrived at another pattern of result if we had used films or clips of films instead of photographs as stimuli. And then prosopagnosia paradox, that is, a preserved semantic memory for faces and a severely impaired episodic memory for faces in SD, would possibly not turn out to be paradoxical at all.

\section{Methodological Issue}

The five SD cases reported in this study were a few years older than the sample of controls who took the Political Faces test. The older control sample did not take this test, and this would have improved the study. However, this relatively younger sample with a mean age of 55 scored at about the same level as the SD cases.

\section{CONCLUSION}

Based on this study and the unsolved methodological issue discussed above we thus propose a follow-up study of face recognition in SD, which would use films or short film clips in addition to photographs as stimuli. This would help to identify the main factor behind the prosopagnosia in SD: time for encoding or lack of action attributes in photos. The critical experiment would be straightforward and demand the following: 1) variation of exposure times of stimuli, and 2) addition of tests of face recognition based on film clips with or without voice. If SD patients perform within the normal range or approach average performance levels at longer exposure times, we may conclude that their atrophy slows down their face encoding. If SD patients perform in the normal range when faces are presented on film, we would be more certain in concluding that their atrophy leads to degraded static information of face processing but leaves activity processing of faces intact.

\section{ACKNOWLEDGEMENTS}

PÖ’s participation was supported by a grant from the Gun and Bertil Stohne Foundation.

\section{REFERENCES}

[1] Crook, T.H. and Larrabee, G.J. (1992) Changes in facial recognition memory across the adult life span. Journal of Gerontology, 47, 138-141. doi:10.1093/geronj/47.3.P138
[2] Measso, G., Romani, L., Martini, E. and Zappala, G. (1990) Preliminary analysis of effects of "normal” aging on different memory processes and abilities. Perceptual \& Motor Skills, 71, 395-401.

[3] Snowden, J.S., Goulding, P.J. and Neary, D. (1989) Semantic dementia: A form of circumscribed cerebral atrophy. Behavioural Neurology, 2, 167-182.

[4] Hodges, J.R., Patterson, K., Oxbury, S. and Funnell, E. (1992) Semantic dementia. Progressive fluent aphasia with temporal lobe atrophy. Brain, 115, 1783-1806. doi:10.1093/brain/115.6.1783

[5] Snowden, J.S., Thompson, J.C. and Neary, D. (2004) Knowledge of famous faces and names in semantic dementia. Brain, 127, 860-872. doi:10.1093/brain/awh099

[6] Simons, J.S., Graham, K.S., Galton, C.J., Patterson, K. and Hodges, J.R. (2001) Semantic knowledge and pisodic memory for faces in semantic dementia. Neuropsychology, 15, 101-114. doi:10.1037/0894-4105.15.1.101

[7] Mondini, S. and Semenza, C. (2006) How Berlusconi keeps his face: A neuropsychological study in a case of semantic dementia. Cortex, 42, 332-335. doi:10.1016/S0010-9452(08)70359-9

[8] Lewin, C. and Herlitz, A. (2002) Sex differences in face recognition-Women's faces make the difference. Brain and Cognition, 50, 121-128. doi:10.1016/S0278-2626(02)00016-7

[9] Julin, P., Almqvist, O., Basun, H., Lannfelt, L., Svensson, L., Winblad, B. and Wahlund, L.O. (1998) Brain volumes and regional cerebral blood flow in carriers of the Swedish Alzheimer amyloid protein mutation. Alzheimer's Disease and Associated Disorders, 12, 49-53. doi:10.1097/00002093-199803000-00008

[10] Neary, D., Snowden, J.S., Gustafsson, L., Passant, U., Stuss, D., Black, S., Freedman, M., Kertesz, A., Robert, P.H., Albert, M., Boone, K., Miller, B.L., Cummings, J. and Benson, D.F. (1998) Frontotemporal lobar degeneration: A consensus on clinical diagnostic criteria. Neurology, 51, 1546-1554. doi:10.1212/WNL.51.6.1546

[11] Jackendoff, R. (1996) The architecture of the linguisticspatial interface. In: Bloom, P., Peterson, M., Nadel, L. and Garrett, M., Eds., Language and Space, MIT Press, Cambridge, 1-30.

[12] Onari, K. and Spatz, H. (1926) Anatomische beitrage zur lehre von der pickschen umschriebenen grosshirnrindenatrophie ("Picksche Krankheit"). (English version: Anatomical contributions to the theory of the circumscribed cortical atrophy of Pick's disease.) Zeitschrift für die Gesamte Neurologie und Psychiatrie, 101, 470-511.

[13] James, T. and Gauthier, I. (2003) Auditory and action semantic feature types activate sensory-specific perceptual brain regions. Current Biology, 13, 1792-1796.

[14] Tyler, L.K., Stamatakis, E.A., Dick, E., Bright, P., Fletcher, P. and Moss, H. (2003) Objects and their actions: Evidence for a neurally distributed semantic system. $\mathrm{Neu}$ roimage, 18, 542-557. doi:10.1016/S1053-8119(02)00047-2

[15] Kable, J.W., Lease-Spellmeyer, J. and Chatterjee, A. (2002) Neural substrates of action event knowledge. Journal of Cognitiuve Neuroscience, 14, 795-805. doi:10.1162/08989290260138681 